\title{
CENTER OF MASS AND KÄHLER STRUCTURES
}

\author{
SCOTT O. WILSON AND MAHMOUD ZEINALIAN
}

\begin{abstract}
There is a sequence of positive numbers $\delta_{2 n}$, such that for any connected $2 n$-dimensional Riemannian manifold $M$, there are two mutually exclusive possibilities:

(1) There is a complex structure on $M$ making it into a Kähler manifold.

(2) For any almost complex structure $J$ compatible with the metric, at every point $p \in M$, there is a smooth loop $\gamma$ at $p$ such that

$$
\operatorname{dist}\left(J_{p}, \operatorname{hol}_{\gamma}^{-1} J_{p} h o l_{\gamma}\right)>\delta_{2 n} .
$$
\end{abstract}

\section{INTRODUCTION}

Kähler manifolds possess a tremendous amount of interesting structure, and therefore have several equivalent characterizations. It has been a focus of much research to determine conditions under which manifolds do (or do not) admit a Kähler structure. This short note shows that the holonomy action on the space of almost hermitian structures determines two mutually exclusive cases, according to whether there is a structure that is nearly preserved at some point, by proving that any manifold with a nearly preserved almost hermitian structure at some point in fact admits a Kähler structure. The novel idea is to use a center of mass argument, averaging a given almost complex structure at a point over the holonomy action, and parallel transporting the result to obtain a global Kähler structure.

Due to the plethora of topological implications for having a Kähler structure, one may deduce several interesting consequences that do not mention the Kähler condition at all. For example, we deduce by [DGMS] that any almost hermitian manifold which has a non-trivial Massey product (or more generally is not formal) also has a holonomy action on the space almost hermitian structures which is bounded away from the identity in a way that is precisely quantifiable.

The authors would like to thank Dennis Sullivan for conversations about this.

2010 Mathematics Subject Classification. 53C55, 32Q15, 53C29.

Key words and phrases. Kähler, almost hermitian, holonomy, center of mass. 


\section{MAIN RESUlT}

For a $2 n$-dimensional real vector space $V$ with an inner product $g$, let

$$
\mathbb{J}(V, g)=\left\{J: V \rightarrow V \mid J^{2}=-i d, g(J u, J v)=g(u, v)\right\}
$$

denote the space of metric almost complex structures on $V$.

Lemma 1. $\mathbb{J}(V, g)$ is a compact smooth manifold. The tangent space at a point $J$ is

$$
T_{J} \mathbb{J}(V, g)=\left\{\phi: V \rightarrow V \mid \phi J=-J \phi \text { and } \phi^{\dagger}=-\phi\right\}
$$

and has a bilinear form $\tilde{g}(\phi, \psi)=\operatorname{tr}\left(\phi \psi^{\dagger}\right)$, for all $\phi, \psi \in T_{J} \mathbb{J}(V, g)$, where $\psi^{\dagger}$ denotes the adjoint of $\psi$. This makes $\mathbb{J}(V, g)$ into a Riemannian manifold on which $O(V)$ acts transitively by isometries.

Proof. $\mathbb{J}(V, g)$ can be identified as the $O(V)$-homogenous space $O(V) / U(V)$ where $U(V)$ is defined using any fixed $J$ on $V$, and the action of $O(V)$ on $\mathbb{J}(V, g)$ is given by conjugation. Therefore $\mathbb{J}(V, g)$ is a smooth manifold. The induced action of $O(V)$ on $T_{J} \mathbb{J}(V, g)$ is also given by conjugation, so that trace is invariant.

Lemma 2. For any two metric vector spaces $(V, g)$ and $(W, h)$ of the same dimension, the Riemannian manifolds $\mathbb{J}(V, g)$ and $\mathbb{J}(W, h)$ are isometric. In particular, for a vector space $V$ and two metrics $g$ and $g^{\prime}$, the Riemannian manifolds $\mathbb{J}(V, g)$ and $\mathbb{J}\left(V, g^{\prime}\right)$ are isometric.

Proof. The Gramm-Schmidt process ensures that there is a linear isometry $f:(V, g) \rightarrow(W, h)$. Conjugation by this isometry gives the desired Riemannian isometry.

For an argument below, we will require convex balls in $\mathbb{J}(V, g)$ for which is there is a well defined notion of center of mass. For any Riemannian manifold $M$, balls of radius $r$ are convex if

$$
r=\min \left\{\frac{\operatorname{inj} M}{2}, \frac{\pi}{2 \sqrt{\epsilon}}\right\},
$$

where inj $M$ denotes the injectivity radius of $M$, and $\epsilon$ is a finite positive upper bound on the sectional curvature (c.f. [Ch] Prop IX.6.1). Also, for any such $\epsilon$, we have the following theorem.

Theorem 3 (Karcher, $[\mathrm{K}]$ ). Let $f: X \rightarrow M$ be a measurable map from a probability space $(X, m)$ to a Riemannian manifold $M$. If $f(X)$ is contained in a convex subset $B$ of $M$, with diameter less than or equal to $\pi / 2 \sqrt{\epsilon}$, then there is a unique center of mass in $B$, defined by the minimum of

$$
E(y)=\frac{1}{2} \int_{X} d^{2}(f(x), y) m(x) .
$$

Additionally, the center of mass is natural with respect to isometries ( $\mathrm{K}$, 1.4.1). We refer the reader to Ch Prop IX.7.1 for an exposition of center of mass. 
Definition 4. For $n>1$ let

$$
\delta_{2 n}=\min \left\{\frac{i n j \mathbb{J}(V, g)}{2}, \frac{\pi}{4 \sqrt{\epsilon}}\right\}
$$

where $V$ is a real vector space of dimension $2 n$ with any metric $g$. Here we choose, once and for all, a finite positive upper bound $\epsilon$ on the sectional curvature of $\mathbb{J}(V, g)$ at one point, which by homogeneity works for all points. By Lemma 2, $\delta_{2 n}$ depends only on the dimension of $V$.

An interesting question (that we will not address here) is whether there is a positive lower bound for the set of all least such $\delta_{2 n}$, independent of $n$.

Definition 5. A complex structure $J \in \mathbb{J}(V, g)$ is said to be nearly preserved by a subgroup $H \subset O(V)$ if the orbit $H J=\left\{\phi^{-1} J \phi \mid \phi \in H\right\}$ lies inside the ball $B\left(J, \delta_{2 n}\right) \subset \mathbb{J}(V, g)$ with center $J$ and radius $\delta_{2 n}$.

Recall that any closed subgroup $H$ of $O(V)$ is a compact Lie group, admitting a bi-invariant Haar measure, which is unique up to a constant. Therefore any such $H$ has a unique probability measure (of total mass equal to one).

Proposition 6. Let $J \in \mathbb{J}(V, g)$ be nearly preserved by a closed subgroup $H$ of $O(V)$. Then there is a $J^{\prime} \in \mathbb{J}(V, g)$ such that $H J^{\prime}=\left\{J^{\prime}\right\}$.

Proof. Consider the orbit $H J \subset B\left(J, \delta_{2 n}\right) \subset \mathbb{J}(V, g)$. By assumption, $B\left(J, \delta_{2 n}\right)$ is a convex ball about $J$. Consider the mapping $H \rightarrow H J \subset \mathbb{J}(V, g)$, from the probability space $H$ onto its orbit. By Definition 4 and Theorem 3 , the set $H J$ has a unique center of mass $J^{\prime}$ in $S$. Since the orbit of the action of $H$ on the set $H J$ is itself, and the center of mass is unique and natural with respect to isometries given by the $H$-action, $H$ fixes $J^{\prime}$.

Given a point $p \in M$, let $H_{p}=\operatorname{Im}\left(\rho_{p}\right)$ be the image subgroup of the holonomy homomorphism $\rho_{p}: \Omega_{p}(M) \rightarrow O\left(T_{p} V\right)$, which is known to be closed by the Ambrose-Singer theorem. The following proposition is a standard result which we include for completeness.

Proposition 7. Let $(M, g)$ be a connected Riemannian manifold and assume $J_{p} \in \mathbb{J}\left(T_{p} M, g\right)$ is invariant under the action of $H_{p}=\operatorname{Im}\left(\rho_{p}\right)$. Then $M$ admits a unique almost complex structure $J: T M \rightarrow T M$ agreeing with $J_{p}$ and making $(M, g, J)$ into a Kähler manifold.

Proof. For any $q \in M$ define $J_{q}: T_{q} M \rightarrow T_{q} M$ by $P_{\lambda} \circ J_{p} \circ P_{\lambda}^{-1}$, where $P_{\lambda}: T_{p} M \rightarrow T_{q} M$ is the Riemannian parallel transport along any smooth path $\lambda$ in $M$ from $p$ to $q$. Since $M$ is connected, such a path $\lambda$ always exists and $J_{q}$ is independent of the choice of the path because $H_{p} J_{p}=\left\{J_{p}\right\}$. Thus, we have defined a smooth complex structure $J: T M \rightarrow T M$, and by way of construction, $J$ is compatible with all parallel transports, and therefore $\nabla J=0$. Since the Levi-Cevita connection $\nabla$ is torsion free, and $J$ is still Hermitian, $J$ is integrable; see Corollary 3.5 of $\underline{\mathrm{KN}}$. Therefore, $(M, g, J)$ is a Kähler manifold. 
Corollary 8. With $(M, g)$ as above, if there is a $p \in M$ and a $J_{p} \in$ $\mathbb{J}\left(T_{p} M, g\right)$ that is nearly preserved by $H_{p}=\operatorname{Im}\left(\rho_{p}\right)$, then there is a $J^{\prime}$ : $T M \rightarrow T M$ making $\left(M, g, J^{\prime}\right)$ into a Kähler manifold.

Proof. This follows from Proposition [6 and Proposition 7.

Theorem 9. There is a sequence of positive numbers $\delta_{2 n} \in \mathbb{R}$ such that for any connected $2 n$-dimensional Riemannian manifold $M$, one of the two following mutually exclusive properties hold:

(1) There is a complex structure on $M$ making it into a Kähler manifold.

(2) For any almost complex structure $J$ compatible with the metric, at every point $p \in M$, there is a smooth loop $\gamma$ at $p$ such that

$$
\operatorname{dist}\left(J_{p}, h o l_{\gamma}^{-1} J_{p} h o l_{\gamma}\right)>\delta_{2 n} \text {. }
$$

Proof. If 1 ) is true then 2) is clearly false, and the converse follows from the previous corollary.

\section{REFERENCES}

[Ch] I. Chavel, Riemannian Geometry: A Modern Introduction, (Cambridge Studies in Advanced Mathematics), $2^{\text {nd }}$ edition.

[DGMS] P. Deligne, P. Griffiths, J. Morgan, D. Sullivan, "Real homotopy theory of Kähler manifolds," Invent. Math. 29 (1975), no. 3, 245-274.

[K] Karcher, H. "Riemannian center of mass and mollifier smoothing," Comm. Pure Appl. Math. 30, (1977), 509-541.

[KN] S. Kobayashi, K. Nomizu, Foundations of Differential Geometry, Vol II, WileyInterscience (1996).

Scott O. Wilson, Department of Mathematics, Queens College, City University of New York, 65-30 Kissena Blvd., Flushing, NY 11367

E-mail address: scott.wilson@qc.cuny.edu

Mahmoud Zeinalian, Department of Mathematics, LiU Post, Long Island University, 720 Northern Boulevard, Brookville, NY 11548, USA

E-mail address: mzeinalian@liu.edu 